\title{
Corrigendum to Impact of Obesity on Short- and Intermediate-Term Outcomes in Inflammatory Bowel Diseases: Pooled Analysis of Placebo Arms of Infliximab Clinical Trials
}

The investigators accidentally omitted acknowledging the role of Yale University Open Data Access Project in making this project feasible. Under the disclosures, the following statement has been added:

"This study, carried out under YODA Project \# 2015-0612, used data obtained from the Yale University Open Data Access Project, which has an agreement with JANSSEN RESEARCH \& DEVELOPMENT, L.L.C.. The interpretation and reporting of research using this data are solely the responsibility of the authors and does not necessarily represent the official views of the Yale University Open Data Access Project or JANSSEN RESEARCH \& DEVELOPMENT, L.L.C..” 